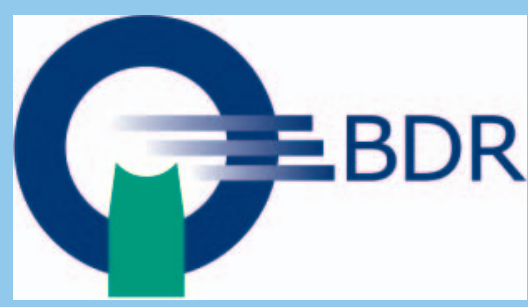

\title{
100. Röntgenkongress - „Radiologie in Klinik und Praxis“
}

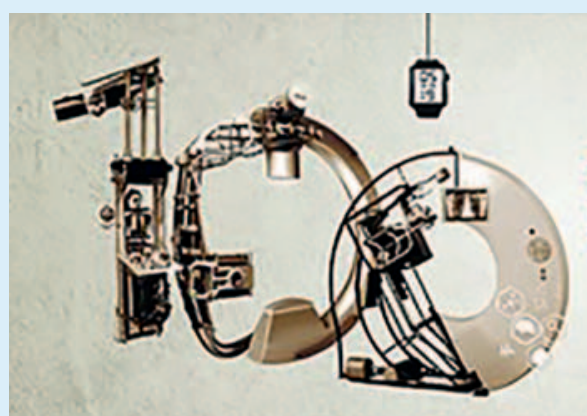

Finanzierung der Radiologie im Krankenhaus: DRG and more

Den Abschluss der berufspolitischen Reihe „Radiologie in Klinik und Praxis“ bildete wie schon im vergangenen Jahr das Seminar „Finanzierung der Radiologie im Krankenhaus - DRG and more" am Samstagvormittag. Bereits zum 3. Mal luden die Moderatoren Prof. Mathias Cohnen, Neuss und Prof. Hermann Helmberger, München im aktuellen Format zu einer Diskussionsrunde im Seminarstil. Die Veranstaltung zu diesem Thema wird wegen seiner Aktualität seit vielen Jahren in modifizierten Formaten angeboten und erfreut sich weiterhin regen Zuspruchs.

In der aktuellen Form führte Achim Schütz, derzeit als Krankenhausgeschäftsführer in Frankfurt tätig, durch das Thema „DRGbasierte und extrabudgetäre Leistungen“. Dabei wurden in interaktiver Diskussion den vor allem jüngeren und damit erst kurz in der Leitungsverantwortung stehenden Kolleg*innen die wesentlichen Grundlagen der DRG-Systematik mit Schwerpunkt Radiologie dargebracht oder repetiert. Zugleich zeigte dieser Teil des Vortrags aber auch Möglichkeiten über den reinen DRG-Bereich hinaus Leistungen zu erbringen und den Deckungsbeitrag der Radiolo- gie zu erhöhen auf. Dies ist allerdings lokal sehr unterschiedlich möglich und muss auf die örtlichen Gegebenheiten angepasst werden.

Im zweiten Teil wurde auf den Grundlagen aufbauend die Diskussion erweitert zum Schwerpunkt „Wie und wo zeigt sich der Mehrwert einer Krankenhausradiologie ggü. partieller oder vollständiger Auslagerung?“. Hierbei standen als zusätzliche Diskussionsteilnehmer zwei erfahrene und mit dem Thema vertraute Radiologen zur Verfügung. Einerseits Prof. Günter Layer, langjähriger Direktor des Zentralinstituts für Diagnostische und Interventionelle Radiologie am Klinikum Ludwigshafen und andererseits Priv.-Doz. Dr. Alexander Stork, der als niedergelassener Radiologe neben den Praxisstandorten zusammen mit seinen Kollegen auch die komplette radiologische Versorgung am Augusta Krankenhaus in Düsseldorf sicherstellt. Ausgehend von den gesetzlichen Rahmenbedingungen entwickelte sich rasch eine Diskussion, die einerseits die Erfahrungen einzelner Teilnehmer mit ihren Geschäftsführungen bzw. Verwaltungsdirektionen, andererseits aber auch die stark lokalen Bedingungen vor Ort als wesentliche Einflussfaktoren aufzeigten. So unterscheiden sich die Gegebenheiten in Ballungsräumen deutlich von denen ländlicher Strukturen. Und das von den Möglichkeiten der Patientenakquise als entscheidendem Steuerungsele- ment ebenso wie der Gewinnung qualifizierten Personals. Einmal mehr ergab die Diskussion, dass die Optimierung der Prozesse in der Radiologie eine wesentliche Voraussetzung für die Wirtschaftlichkeit darstellt. Die Optimierung der Abläufe im übrigen Klinikum sind jedoch mindestens ebenso wichtig für einen wirkungsvollen Einsatz der technischen Ressourcen unseres Faches.

Zusammenfassend haben die Teilnehmer das Seminar gerüstet mit einer guten Basis für die Diskussion im heimischen Umfeld verlassen. Erweitert um die Erkenntnis, dass das Engagement lokal mit der eigenen Geschäftsführung entscheidend für den Erfolg ist, es keine bundeseinheitlichen Erfolgsrezepte gibt und die lokalen Gegebenheiten auch über die Strukturen der Radiologie im eigenen Krankenhaus entscheiden werden. Kooperationen sind über die Sektoren hinweg möglich, abhängig von den örtlichen Verhältnissen sinnvoll und fruchtbar, aber es nicht in allen Fällen die für die Versorgung der Patienten geeignetste Organisationsform ist. Die Veränderungen, die sich in unserem Fach sowohl in medizinischer als auch in wirtschaftlicher Form abzeichnen, lassen es jedoch angeraten sein, sich intensiv mit dem Thema zu beschäftigen, den Blick zu weiten für neue Optionen und prinzipiell offen an moderne Organisationsformen heranzugehen. Immer unter der Prämisse der besten Versorgung unserer Patienten und in einem fairen Miteinander der beteiligten Partner.

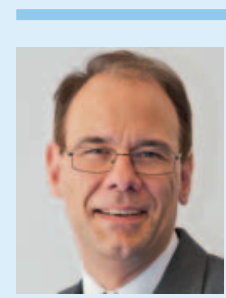
Prof. Hermann Helmberger München 